\title{
Myelofibrosis in Patients of Chronic Myeloid Leukemia in Chronic Phase at Presentation
}

Ambareen Hamid1, Sobia Ashraf1, Samina Qamar1, Muhammad Asif Naveed1, Ahmad Hameed1 and Muhammad Azhar Farooq² Department of Pathologyl / Paediatric Medicine2, King Edward Medical University (KEMU), Lahore, Pakistan

\begin{abstract}
Objective: To determine the frequency and grading of myelofibrosis in patients of chronic myeloid leukaemia in chronic phase, and the association of cytopenias/cytosis with the degree of fibrosis.

Study Design: Descriptive study.

Place and Duration of Study: King Edward Medical University, Lahore, from March 2015 to April 2017.

Methodology: Patients of both genders and all age groups with Philadelphia chromosome/BCR-ABL positivity were included in the study. Detailed medical history and examination findings were recorded. Sample for CBC was taken in EDTA and run on automated haematology analyser. Bone marrow aspirate and trephine biopsy was done from posterior iliac crest. Bone marrow aspirate slides were stained with Giemsa stain. The trephine biopsy was processed and stained with haematoxilyn and eosin. Silver (reticulin) and trichrome staining was done on trephine biopsy to assess the degree of fibrosis. Grading of fibrosis was done according to WHO revised classification 2016.

Results: Among the 82 cases, $65 \%(n=53)$ were having WHO Grade MF 1 myelofibrosis, $27 \%(n=22)$ WHO Grade MF 2 myelofibrosis; whereas, $08 \%(n=07)$ WHO Grade MF 3 myelofibrosis. CBC counts were analysed to see any association between anemia, thrombocytocytosis and leucocytosis with advanced fibrosis ( $p=0.148,0.413$ and 0.174 , respectively).

Conclusion: Some degree of bone marrow fibrosis was present in all patients of chronic myeloid leukemia, while advance fibrosis (WHO Grade MF 2 and 3 ) is also common. Peripheral blood counts are not predictor of increased fibrosis. Therefore, bone marrow biopsy with special staining should be done in all cases of chronic phase of CML.
\end{abstract}

Key Words: Chronic myeloid leukemia, Myelofibrosis, Cytopenia, Cytosis eosin, hemotoxylin, WHO revised classification 2016, Blood counts, Anemia, Leucocytosis, Thrombocytosis, Special staining.

How to cite this article: Hamid A, Ashraf S, Qamar S, Naveed MA, Hameed A, Farooq MA. Myelofibrosis in patients of chronic myeloid leukemia in chronic phase at presentation. J Coll Physicians Surg Pak 2019; 29(11):1096-100.

\section{INTRODUCTION}

Chronic myeloid leukaemia is a chronic clonal myeloproliferative disorder. The disease is characterised by an inappropriate increase in granulocytic series cells. ${ }^{1}$ It is caused by reciprocal balanced translocation between chromosome 9 and 22, t $(9,22)$ (q34; q11), leading to formation of Philadelphia chromosome. The resulting $B C R-A B L 1$ fusion gene leads to formation of BCR-ABL oncoprotein with enhanced tyrosine kinase activity. This unprovoked tyrosine kinase activity is responsible for morphological and clinical features of chronic myeloid leukaemia. ${ }^{2}$ The incidence is $1-2 / 100000.3$ The disease is characterised usually by three phases: chronic phase, accelerated phase, and blastic phase; but sometimes, it can be biphasic skipping the accelerated phase. 4 Majority of patients are diagnosed in chronic phase in which there is raised TLC (>11,000/microlitre), normal or raised platelet count on $\mathrm{CBC}$ examination, and hypercellular marrow with normal or slightly left shifted

Correspondence to: Dr. Sobia Ashraf, Department of Pathology,

King Edward Medical University (KEMU), Lahore, Pakistan

E-mail: s.qayyum81@gmail.com

Received: November 27, 2018; Revised: March 19, 2019

Accepted: April 22, 2019 myeloid maturation. In accelerated phase, leukocytosis is usually accompanied by increase in myeloblasts, basophilia, thrombocytopenia or thrombocytosis; and progressive splenomegaly is seen even with treatment. Clonal evolution leading to cytogenetic abnormalities other than the Philadelphia chromosome are also seen. In the third, i.e. blast phase, blast count rapidly rises and marrow failure occurs. The morphological picture at this stage resembles that of acute leukaemia.5,6

Myelofibrosis, seen in CML, is characterised initially by increased number of reticulin fibres and then collagen fibres. It occurs due to cytokine release and activation of fibroblasts. In the early stage of myelofibrosis in chronic phase $\mathrm{CML}$, atypical small mega karyocytes secrete PDGF, possibly PDGF-AB, along with other growth factors. As the disease progresses to accelerated or blast phase, a larger quantity of PDGF-AB or PDGF-BB is released from blast cells. In addition, some fibroblasts are attracted by the PDGF and multiply, and deposit collagen as well as fibronectin in the bone marrow stroma. ${ }^{7}$ Increased reticulin and collagen fibrosis is seen in all phases of disease and has been considered as important prognostic marker. In the beginning of imatinib therapy, one study had negated its significance; 8 but recent studies have again emphasised this fact that 
grading of fibrosis has played a significant role in morbidity and mortality related to CML. ${ }^{9}$

Fibrosis is best identified by using special stains (silver stain for reticulin and trichrome stain for collagen). ${ }^{10}$ It is graded according to WHO guidelines based on extent of fibrosis. In majority of the studies done so far, dense fibrosis was seen mostly in accelerated phase. In this study, we have aimed to find patterns of fibrosis in CML in chronic phase at the time of presentation. This will highlight the significance of bone marrow biopsy and will identify patients with advanced fibrosis at the time of presentation so that their treatment can be modified, accordingly.

The objective of the study was to determine the frequency and grading of myelofibrosis in patients of chronic myeloid leukaemia in chronic phase, and the association of cytopenias/cytosis (anemia, thrombocytopenia, thrombocytosis and leukocytosis) with the degree of fibrosis.

\section{METHODOLOGY}

It was a descriptive cross-sectional study, carried out in King Edward Medical University, Lahore, from March 2015 to April 2017. Inclusion criteria were patients of both genders and all age groups with Philadelphia chromosome/BCR- ABL positivity and in chronic phase. Exclusion criteria were patients of CML getting treatment, in accelerated phase or blast phase. After informed consent, a detailed medical and personal history was taken. Peripheral venous blood in EDTA for complete counts was drawn and was run on automated haematology analyser. Manual verification was done for high white blood cell and platelet counts. Bone marrow aspirate and trephine biopsy was done from posterior iliac crest. Bone marrow aspirate slides were stained with Giemsa stain. The trephine biopsy was processed in standardised manner and stained with hematoxylin and eosin. Silver and trichrome staining was done on trephine biopsy to assess the degree of fibrosis. Bone marrow fibrosis might be a hidden condition in CML patients, which may be negative due to the use of H\&E stain only. ${ }^{10}$ Grading of fibrosis was done according to WHO revised classification 2016. 11

SPSS version 21 was used to analyse the data. Frequencies and grades of myelofibrosis were determined. Qualitative variables, i.e cytopenias/cytosis (anemia, thrombocytosis and leukocytosis) were analysed to determine their association with grades of fibrosis using descriptive statistics and crosstabs, keeping the confidence interval of $95 \%$ and $p$-value of $\leq 0.05$.

\section{RESULTS}

A total of 82 cases were included in the study. Out of these, $44(53.6 \%)$ were males and $38(46.4 \%)$ were females. Age group range was from 11 to 80 years.
Table I: Frequency and severity of fibrosis in different age groups.

\begin{tabular}{l|c|l|l|c}
\hline & \multicolumn{3}{|c|}{ Fibrosis } & Total \\
\cline { 2 - 4 } & Grade 1 & Grade 2 & Grade 3 & \\
\hline Age & & & & \\
$11-20$ & $3(3.7 \%)$ & $1(1.2 \%)$ & $1(1.2 \%)$ & $5(6.1 \%)$ \\
$21-30$ & $17(20.7 \%)$ & $7(8.5 \%)$ & $1(1.2 \%)$ & $25(30.4 \%)$ \\
$31-40$ & $13(15.8 \%)$ & $4(4.8 \%)$ & $2(2.4 \%)$ & $19(23.1 \%)$ \\
$41-50$ & $11(13.4 \%)$ & $9(11 \%)$ & $2(2.4 \%)$ & $22(26.8 \%)$ \\
$51-60$ & $7(8.5 \%)$ & 0 & 0 & $7(8.5 \%)$ \\
$61-70$ & $1(1.2 \%)$ & 0 & 0 & $1(1.2 \%)$ \\
$71-80$ & $1(1.2 \%)$ & $1(1.2 \%)$ & $1(1.2 \%)$ & $3(3.7 \%)$ \\
\hline Total & $53(65 \%)$ & $22(27 \%)$ & $7(8.5 \%)$ & 82 \\
\hline
\end{tabular}

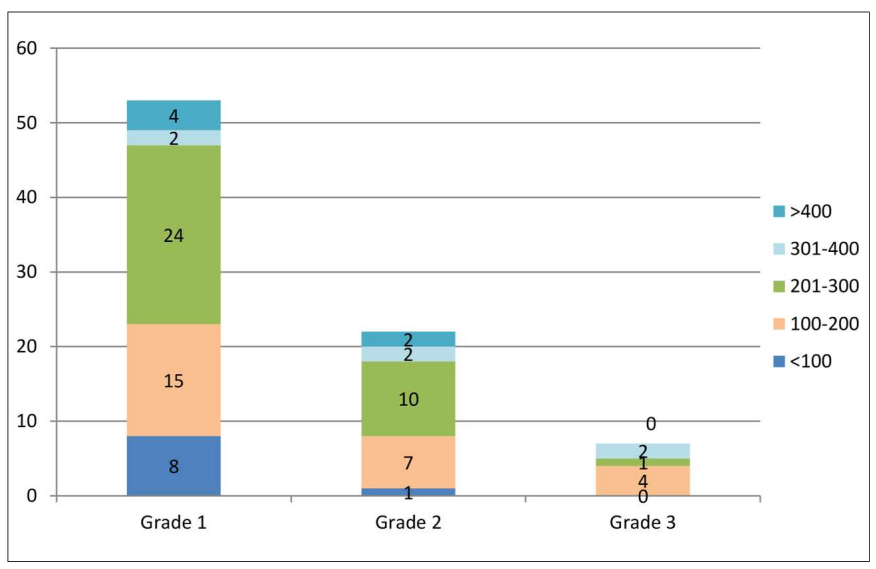

Figure 1: White cell count (x109/L) in different fibrosis grades of $C M L(n)$.

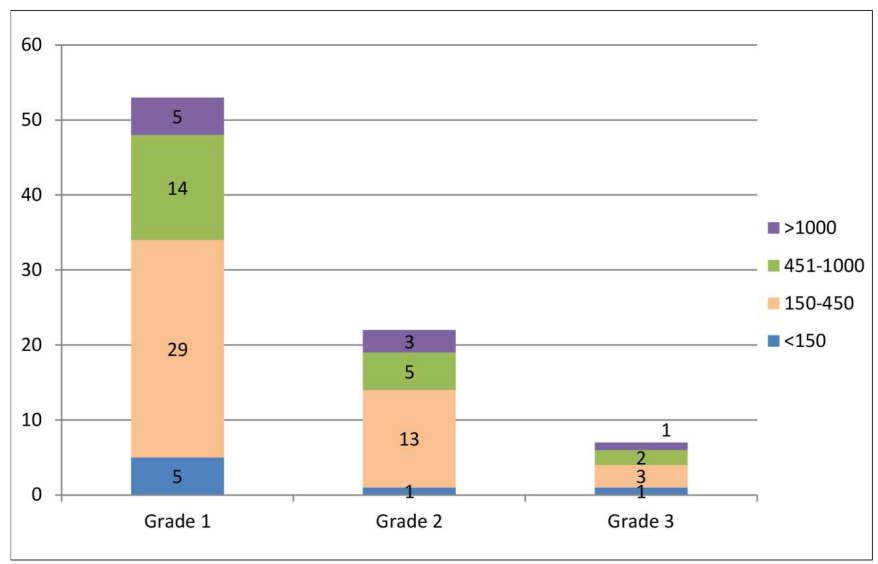

Figure 2: Patient count $(x 109 / L)$ in different grades of $C M L(n)$.

Majority of patients belonged to age group from 21-30 years (Table I). Fibrosis of varying degree was seen in all patients. Grade 1 fibrosis was seen in 53 (65\%) patients, grade 2 in $22(27 \%)$ patients and grade 3 fibrosis was present in $7(8 \%)$ patients. The cross tabulation between age and severity of fibrosis is given in Table I.

$\mathrm{CBC}$ counts were analysed to assess any association between anemia, thrombocytosis and leucoytosis with advanced fibrosis. There was no significant association of these abnormalities with advanced fibrosis $(p=0.148$, 0.413 and 0.174 , respectively, Figures 1 and 2). 


\section{DISCUSSION}

Chronic myeloid leukaemia is a clonal malignancy and is uncontrolled proliferation of myeloid series cells and megakaryocytes leading to bone marrow fibrosis.12,13 This study included the patients of chronic myeloid leukaemia, in chronic phase at presentation with positive BCR-ABL fusion gene. In this study, a total of 82 patients were included. Majority of them $(n=44)$ were males. Females were in lower proportion $(n=38)$. Similar findings were also reported by Bhatti et al. ${ }^{14}$

Majority of our patients $(n=66)$ were in the age group between $21-50$ years $(80.5 \%)$ and in this group peak incidence is in $21-30$ years group. The study conducted by Bhatti et al. in Northern Pakistan and by Chang et al. in Sindh has shown peak age in the same group and is in accordance with this study.13,14 The data from UK and USA has shown peak incidence in older age group. ${ }^{15,16}$ Bhatti et al. attributed this fact to demographic differences between nations; in the present report, there were greater number of younger subjects and a much smaller number of subjects beyond 60 years.

Mild to severe fibrosis was seen in all patients of CML. In this study, $65 \%$ out of 82 patients $(n=53)$ had mild (grade 1) fibrosis. While grade 2 and grade 3 fibrosis was seen in $35 \% \quad(n=29)$. In a study conducted by Kantarjian et al. on 198 patients, severe bone marrow fibrosis was seen in $38 \%$ cases. ${ }^{17}$ While Tanrikulu et al. found severe bone marrow fibrosis in $33 \%$ of patients of CML at presentation. ${ }^{18}$ The findings of this study are in concordance with the studies conducted internationally.

In chronic stage, grade 2 and 3 fibrosis was also seen in addition to grade1. In this study, it was seen that grade 2 and 3 fibrosis was more common in younger age group (11-50 years) as compared to older age group (51-80 years). In younger age group, 27 out of $71(38 \%)$ had grade 2 and 3 fibrosis while in older age group 2 out of $11(18 \%)$ patients had advanced fibrosis. Advanced stage is a sign of progression of disease; and if present at initial presentation then it is suggestive of aggressive disease. 19 These facts indicate that CML is more aggressive in younger age group as compared to older age group. A cohort study conducted by Castagnetti et al. in 2014 on 2,784 patients of CML has analysed this fact. They have studied differences in spleen size, blood counts, Sokal and EUTOS score in young adults, adults and elderly over period of 40 years. They clearly demonstrated that clinical presentation, disease progression and response to tyrosine kinase inhibitors are more severe in young adults. ${ }^{19} \mathrm{Hijiya}$ et al. have suggested that pediatric CML is more aggressive than adult CML; and there are genetic differences among two groups so the children should be offered more definite treatment to avoid long term complications of TKI drugs. ${ }^{20}$

In this study, observations regarding different haematological parameters and degree of fibrosis were also made. Anaemia was observed in $71 \%$ of cases; and $44 \%$ were in grade 1 fibrosis. Anemia was not associated $(p=0.148)$ with advanced fibrosis as majority of the patients with anemia had grade 1 fibrosis. Saleem et al. found anaemia more with advanced fibrosis. ${ }^{21}$ In that study, patients of all phases were included; whereas, the present authors included only chronic phase and patients in accelerated and advanced phases were excluded.

In this study, high leucocyte count was not observed with increased degree of fibrosis, (Figure 1, $p=0.413$ ). This is in concordance with study conducted by Saleem et al., who did not find rising leucocyte count with increased degree of fibrosis. ${ }^{21}$

In this study, $54 \%$ patients had platelet count in normal range $(150,000-400,000 / u l)$, majority of them $(35 \%)$ were in grade 1 fibrosis (Figure 2, $p=0.174$ ). The finding is supported by a study conducted by Ernesto Vigna who did not find any significant association between rising platelet count and fibrosis. ${ }^{22}$

Above findings indicate that anemia, leukocytosis and thrombocytosis are not indicative of advanced fibrosis, and trephine biopsy is essential in each case to determine degree of fibrosis. It has been considered as an independent prognostic marker as it played a significant role in morbidity and mortality related to CML as explained by Hussein et al. 23

Myelofibrosis is an ominous sign and impairs drug penetration and can affect outcomes. 24 A study conducted by Kantarijan et al. in 2005 compared the results of imatinib therapy on patients with mild to moderate, and those with severe fibrosis. He observed similar response, complete cytogenetic response, in two groups and failure-free survival were also same. He proposed that bone marrow fibrosis was not prognostic marker in CML in the era of imatinib therapy. ${ }^{7}$

In another study by Kantarijian et al., effects of fibrosis on prognosis of patients in chronic phase of CML treated with imatinib were noted. It was seen that advanced fibrosis improved with the therapy. However, $15 \%$ still had worse outcome.18 A study by Buesche et al. conducted over a period of 5 years on 59 patients of CML showed that pretreatment fibrosis was bad prognostic sign, Imatinib therapy reduced and reversed the degree of fibrosis, but in longer follow up period, many patients developed severe fibrosis. ${ }^{7}$ They stated that imatinib therapy was not effective in eliminating unfavourable prognosis of MF and did not offer complete cytogenetic response in all patients with fibrosis.

A study done by Eliacik assessed the pretreatment and posttreatment fibrosis and dysplasia in CML patients receiving first generation (imatinib) or second generation (dasatinib and nilotinib) tyrosine kinase inhibitors (TKI). ${ }^{25} \mathrm{He}$ suggested that bone marrow fibrosis was an independent marker of prognosis and indicator of 
response to TKI drugs in CML. The author emphasised that fibrosis should be considered as important and critical parameter regarding decision of treatment.

All above data favours myelofibrosis as an important prognostic indicator of CML; and its detection at the time of diagnosis helps in decisions regarding treatment of the patient.

\section{CONCLUSION}

Bone marrow fibrosis is seen in all patients of chronic myeloid lekemia; and among these cases, advanced fibrosis are also seen. Peripheral blood counts are not predictor of increased fibrosis; therefore, bone marrow biopsy with special staining should be done in all cases of chronic phase of CML.

\section{PATIENTS' CONSENT:}

Informed consents were taken from the patients.

\section{CONFLICT OF INTEREST:}

Authors declared no conflict of interest.

\section{AUTHORS' CONTRIBUTION:}

$\mathrm{AH}$ : Topic selection, review and correction of final manuscript.

SA: Introduction writing, discussion writing, correspondence.

SQ: Data collection and literature review.

MAN: Data analysis.

$\mathrm{AH}$ : Data collection and results formulation.

MAF: Data collection.

\section{REFERENCES}

1. Savage D, Szydlo RM, Goldman JM. Clinical features at diagnosis in 430 patients with chronic myeloid leukaemia seen at a referral centre over a 16-year period. Br J Haematol 1997; 96:111-6.

2. Swerdlow SH, Campo C, Harris NL, Jaffe ES, Pileri SA, Stein $\mathrm{H}$, et al. WHO classification of tumours of haematopoietic and lymphoid tissues. IARC Press: Lyon, France, 2008.

3. Huang $\mathrm{X}$, Cortes $\mathrm{J}$, Kantarjian $\mathrm{H}$. Estimations of the increasing prevalence and plateau prevalence of chronic myeloid leukemia in the era of tyrosine kinase inhibitor therapy. Cancer 2012; 118:3123-7.

4. Vardiman JW. Chronic myelogenous leukemia, BCR-ABL1+. Am J Clin Pathol 2009; 132:250-60.

5. Thompson PA, Kantarjian HM, Cortes JE. Diagnosis and treatment of chronic myeloid leukemia in 2015. Mayo Clin Proc 2015; 90:1440-54.

6. Shuai K, Halpern J, ten Hoeve J, Rao X, Sawyers CL. Constitutive activation of STAT5 by the BCR-ABL oncogene in chronic myelogenous leukemia. Oncogene 1996; 13:247-54.

7. Buesche G, Ganser A, Schlegelberger B, von Neuhoff N, Gadzicki D, Hecker $\mathrm{H}$, et al. Marrow fibrosis and its relevance during imatinib treatment of chronic myeloid leukemia. Leukemia 2007; 21:2420-7.
8. Kantarjian HM, Bueso-Ramos CE, Talpaz M, O'Brien S, Giles F, Rios MB, et al. The degree of bone marrow fibrosis in chronic myelogenous leukemia is not a prognostic factor with imatinib mesylate therapy. Leuk Lymphoma 2005; 46:993-7.

9. Pandey N, Yadav G, Kushwaha R, Verma SP, Singh US, Kumar A, et al. Effect of imatinib on bone marrow morphology and angiogenesis in chronic myeloid leukemia. Adv Hematol 2019; 2019:1835091.

10. Al-Khafaji AKI, Al-Shammari HHJ, Al-Obeidi SRH. Bone marrow fibrosis in chronic myeloid leukemia (CML) and other myeloproliferative disorders evaluated by using special histochemical stains for collagen. J Fac Med Baghdad 2011; 53:296-300.

11. Arber DA, Orazi A, Hasserjian R, Thiele J, Borowitz MJ, Le Beau MM, et al. The 2016 revision to the World Health Organization classification of myeloid neoplasms and acute leukemia. Blood 2016; 127:2391-405.

12. Thiele J, Kvasnicka HM, Schmitt-Graeff A, Zirbes TK, Birnbaum F, Kressmann C, et al. Bone marrow features and clinical findings in chronic myeloid leukemia - a comparative, multicenter, immunohistological and morphometric study on 614 patients. Leuk Lymphoma 2000; 36:295-308.

13. Bhatti FA, Ahmed S, Ali N. Clinical and haematological features of 335 patients of chronic myelogenous leukaemia diagnosed at single center in Northern Pakistan. Clinical medicine insight: Blood disorders 2012; 5:15-24.

14. Chang F, Qazi RA, Khan M, Baloch S, Sahito MM, Mir A. Clinico hematological profile and phase distribution of chronic myeloid leukemia. Biol Med (Aligarh) 2015; 7:5.

15. National Cancer Institute. Surveillance Epidemiology and End Results (SEER Stat Fact Sheet: Chronic Myeloid Leukemia) [online] 1975-2009 (Vintage 2009 populations) (Released Apr 16, 2012). Available from: http://www.seer.gov/statfacts/html/ cmyl.html\#incidence-mortality.

16. Bhayat F, Das-Gupta E, Chris Smith, McKeever T, Hubbard R. The incidence of and mortality from leukemias in the UK: A general population-based study. BMC Cancer 2009; 9:252.

17. Kantarjian HM, Bueso-Ramos CE, Talpaz M, O'Brien S, Giles F, Faderl S, et al. Significance of myelofibrosis in early chronicphase, chronic myelogenous leukemia on imatinib mesylate therapy. Cancer 2005; 104:777-80.

18. Simsek ET, Eskazan AE, Cengiz M, Cem Ar M, Ekizoglu S, Salihoglu $A$, et al. Imatinib mesylate reduces bone marrow fibrosis and overwhelms the adverse prognostic impact of reticulin formation in patients with chronic myeloid leukemia. Blood 2015; 126:2783.

19. Castagnetti F, Gugliotta G, Baccarani M, Breccia M, Specchia G, Levato $\mathrm{L}$, et al. Differences among young adults, adults and elderly chronic myeloid leukemia patients. Ann Oncol 2015; 26:185-92.

20. Hijiya N, Schultz KR, Metzler M, Millot F, Suttorp M. Pediatric $\mathrm{CML}$ is a unique disease that requires a different approach. Blood 2016; 127:392-9.

21. Saleem M, Kumar A, Tripathi A, Singh US, Kushwaha MRS. Platelets, megakaryocytes and marrow fibrosis: A prospective study in chronic myeloid leukemia. J Med Erudition. 2003; 01:1-11.

22. Vigna E, Martino B, Bacci F, Recchia AG, Mendicino F, Morelli R, 
et al. Disappearance of bone marrow fibrosis in apatient with chronic myeloid leukemia treated with dasatinib. Chemo-therapy $2017 ; 62: 350-2$.

23. Dekmezian R, Kantarjian HM, Keating MJ, Talpaz M, McCredie $\mathrm{KB}$, Freireich EJ. The relevance of reticulin stain measured fibrosis at diagnosis in chronic myelogenous leukemia. Cancer 1987; 59:1739-43.

24. Hussein K, Stucki-Koch A, Göhring G, Kreipe H, Suttorp M. Increased megakaryocytic proliferation, pro-platelet deposition and expression of fibrosis-associated factors in children with chronic myeloid leukaemia with bone marrow fibrosis. Leukemia 2017; 31:1540-6.

25. Eliacik E, Isik A, Aydin C, Uner A, Aksu S, Sayinalp N, et al. Bone marrow fibrosis may be an effective independent predictor of the 'TKI drug response level' in chronic myeloid leukemia. Hematology 2015; 20:392-6.

......... 